Materials and Methods We screened $\mathrm{T}$ cell activation and exhaustion markers, among others, on different tumor tissues using the MACSima ${ }^{\mathrm{TM}}$ Imaging Platform, an instrument for the highly multiplexed immunofluorescence imaging technology MICS (Multiparameter Imaging Cell Screen), enabling investigation of hundreds of markers on a single section. Moreover, flow cytometry and single-cell RNA sequencing analyses of T cells from tumor digests were performed to complement the characterization of TILs.

Results The MICS results highlighted the complexity of the TME, mainly composed of tumor cells, fibroblasts and endothelial vessels. In some cases, an extensive immune infiltrate consisted of $\mathrm{T}$ cells, plasma cells, some $\mathrm{B}$ cells and distinct myeloid cells was observed. Particularly, CD8 T cells from different tumor areas exhibited a tissue-resident memory phenotype with the expression of CD69, CD45RO or CD103. Activated/exhausted CD8 T cells were homogenously found across the imaged tumor areas. However, there was a tendency to find them in close proximity to tumor cells, especially for CD8 subsets expressing CD39 and other relevant markers, which may suggest the identification of tumor-reactive CD8 T cell populations. Flow cytometry data revealed the presence of similar $\mathrm{T}$ cell phenotypes in the patient's TILs from tumor digests.

Conclusions This imaging technology offers the possibility to study multiple parameters - including the localization-of relevant cells in the TME such as T cells. The phenotypic and functional characterization of different $\mathrm{T}$ cell subsets will allow the further investigation of their anti-tumor reactivity. Ultimately, the enrichment and expansion of the identified tumorreactive $\mathrm{T}$ cell population hold great promises to improve the efficiency of $\mathrm{T}$ cell therapy against cancer.

Disclosure Information E. Criado-Moronati: A. Employment (full or part-time); Significant; Miltenyi Biotec B.V. \& Co. KG. A. Gosselink: A. Employment (full or part-time); Significant; Miltenyi Biotec B.V. \& Co. KG. J. Kollet: A. Employment (full or part-time); Significant; Miltenyi Biotec B.V. \& Co. KG. A. Dzionek: A. Employment (full or part-time); Significant; Miltenyi Biotec B.V. \& Co. KG. B. Heemskerk: A. Employment (full or part-time); Significant; Miltenyi Biotec B. V. \& Co. KG.

\section{P06.15 HIGHLY MULTIPLEXED, SINGLE-CELL FUNCTIONAL PROTEOMICS OF CAR-T PRODUCTS ENABLES MORE PREDICTIVE PRODUCT CHARACTERIZATION, CELL MANUFACTURING OPTIMIZATION, AND CELLULAR BIOMARKERS ACROSS PRODUCT TYPES}

D Liu*, P Paczkowski, S Mackay, J Zhou. IsPlexis, Branford, CT, USA

\subsection{6/jitc-2020-ITOC7.94}

Chimeric antigen receptor (CAR) $\mathrm{T}$ cell therapy has already paved the way for successful immunotherapies to fight against liquid tumors and is quickly expanding to solid tumors. Nevertheless, the biggest challenges are how to evaluate the quality of CAR-T cells and how to predict their in vivo behaviors once reinfused into a patient. In this report, we review single-cell polyfunctional profiling results obtained from several different sets of pre-infusion CAR-T samples, including CD19 CAR-T products from Novartis and Kite Pharma (Gilead), GoCAR-T cell products targeting Prostate Stem Cell Antigen from Bellicum, bispecific CD19/22 CAR-T cells from the NIH, trimeric APRIL-based CAR-T cells targeting both BCMA and TACI from MGH and CAR-T cells targeting glypican 3 in hepatocellular carcinoma from NIH. In each case, CD4+ and CD8 + CAR-T cells were stimulated and subsequently analyzed at a single-cell level using IsoPlexis' IsoCode proteomic chips. Our single-cell data revealed highly polyfunctional and heterogeneous responses across each cohorts. The polyfunctional strength index (PSI) of the pre-infused CAR-T products is significantly associated with the clinical outcome of the patients after receiving the treatment, as well as post-infusion grade 3 + CRS. The CAR-T cells secreted a wide range of cytokines/ chemokines in response to antigen specific stimulation and a significant portion of the CAR-T cells were polyfunctional (2 + cytokines/cell). These results highlight the potential benefits of single-cell proteomics to comprehensively understand how CAR-T products behave in response to antigen-specific stimulation. Analyzing the single-cell polyfunctionality of CAR-T profiles also provides a valuable quality check for optimizing the manufacturing process and a powerful tool for next generation biomarker developments.

Disclosure Information D. Liu: None. P. Paczkowski: None. S. MacKay: None. J. Zhou: None.

\section{P07 Cell Therapy in Haematologic Diseases}

\section{P07.01 CD19 CAR T-CELLS FOR RELAPSED/REFRACTORY DIFFUSE LARGE B-CELL LYMPHOMA: REAL-WORLD DATA FROM LMU MUNICH}

\begin{abstract}
1,2V Bücklein*, 1,2V Blumenberg, ${ }^{1} \mathrm{C}$ Schmidt, ${ }^{1,2} \mathrm{~K}$ Rejeski, 1,2M Ruzicka, ${ }^{1,2} \mathrm{~N}$ Müller, $1,{ }^{2} \mathrm{~A}$ Reischer, ${ }^{3} \mathrm{~L}$ von Baumgarten, ${ }^{1} \mathrm{~A}$ Völkl, ${ }^{4} \mathrm{~B}$ Wagner, ${ }^{4} \mathrm{~A}$ Humpe, ${ }^{1} \mathrm{~J}$ Tischer, ${ }^{1} \mathrm{H}$ Stemmler, ${ }^{1,5} \mathrm{M}$ von Bergwelt, ${ }^{1,2,5} \mathrm{M}$ Subklewe. ${ }^{1}$ Department of Medicine III, University Hospital, LMU Munich, Munich, Germany; '2Laboratory for Translational Cancer Immunology, LMU Gene Center, Munich, Germany; ${ }^{3}$ Department of Neurology, University Hospital, LMU Munich, Munich, Germany; ${ }^{4}$ Department of Transfusion Medicine, University Hospital, LMU Munich, Munich, Germany; ${ }^{5}$ German Cancer Consortium (DKTK) and German Cancer Research Center, Heidelberg, Germany
\end{abstract}

\subsection{6/jitc-2020-ITOC7.95}

Background The anti-CD19 CAR T-cell products Axicabtagene Ciloleucel (Axi-cel) and Tisagenlecleucel have been approved by the EMA for the treatment of patients (pts) with relapse/ refractory $(r / r)$ diffuse large B-cell lymphoma (DLBCL) in August 2018. In clinical trials, both cell products induced ongoing complete responses in heavily pretreated patients. However, this activity was associated with significant toxicity. We evaluated the outcomes of DLBCL pts treated with Axi-cel and Tisagenlecleucel at the LMU Munich.

Materials and Methods CAR T cell product characteristics, toxicity and response rates of pts treated at our center between January and October 2019 were retrospectively assessed.

Results As of October 2019, 24 out of 34 r/r DLBCL pts (71\%) with confirmed CAR $\mathrm{T}$ cell treatment indication were leukapheresed. Four apheresed pts died before CAR T cell therapy due to rapidly progressive disease. So far, 17 DLBCL pts have been treated. Median age was 60 years (range 1974). ECOG was $0-1$ in eleven, and $2-3$ in six pts. Eight pts had undergone prior stem cell transplant (6 autologous, 2 allogeneic SCT). 13 pts received bridging chemotherapy between leukapheresis and CAR T cell transfusion. Only 6 (35\%) of the 17 transfused pts would have met the inclusion criteria of the pivotal clinical trials (JULIET, ZUMA-1). 